\title{
The Prevalence of Reproductive Tract Infections in a Chinese Internal Migrant Population, and Its Correlation with Knowledge, Attitude, and Practices: A Cross-Sectional Study
}

\author{
Shuangfei $\mathrm{Xu}^{1,2,3,+}$, Chuanning Yu${ }^{4,+}$, Ying Zhou ${ }^{1,2} \oplus^{\circ}$, Junqing $\mathrm{Wu}^{1,2}$, Tieling Bai ${ }^{5}$, \\ Junxian Zhang ${ }^{6}$ and Yuyan $\mathrm{Li}^{1,2, *(\mathbb{D})}$ \\ 1 Key Lab of Reproduction Regulation of National Population and Family Planning Commission, \\ Shanghai Institute of Planned Parenthood Research, Institute of Reproductive Development, \\ Fudan University, Shanghai 210035, China; 15211150007@fudan.edu.cn (S.X.); \\ yingzhou2012@163.com (Y.Z.); wujq1688@163.com (J.W.) \\ 2 School of Public Health, Fudan University, Shanghai 200032, China \\ 3 Zhejiang Provincial Center for Disease Control and Prevention, Hangzhou 310051, China \\ 4 Longhua District Center for Chronic Disease Control (Mental Health Center), Shenzhen 518110, China; \\ yu.chuanning@icloud.com \\ 5 Family Planning and Reproductive Health Instructing Center of Ningxia Hui Autonomous Region, \\ Yinchuan 750000, China; luxn001@163.com \\ 6 Family Planning Publicity and Education Technical Advising Center of Urumchi, Urumchi 830092, China; \\ junxianzhang@sina.cn \\ * Correspondence: liyuyan@sippr.org.cn; Tel.: +86-21-64771670 \\ + Shuangfei Xu and Chuanning Yu contributed equally.
}

Received: 4 January 2019; Accepted: 18 February 2019; Published: 22 February 2019

check for updates

\begin{abstract}
Objective: This study was designed to assess the prevalence of reproductive tract infections (RTIs) among an internal-migrant population of reproductive age in China. We also analyzed the knowledge, attitude, and practices related to these infections. Methods: A cross-sectional study using the quota-sampling method was conducted in three cities from March 2016 to February 2017. A total of 3320 participants (40.7\% men and 59.3\% women) were enrolled in the study, of whom, 1124, 1015, and 1181 were from Yinchuan, Urumchi, and Shanghai, respectively. Data on the included subjects were collected using a self-administered questionnaire. Results: We found that $3.2 \%$ and $22.6 \%$ of all subjects lacked knowledge regarding the identification and prevention of RTIs, respectively. More than $80 \%$ of the participants approved of developing RTI surveillance and taking RTI-related courses. While $45.1 \%$ of the respondents changed their underwear every $2-3$ days, $49.0 \%$ cleaned their genitals daily, and 34.9\% reported taking a bath daily. Among contraceptive users, $47.4 \%$ and $29.7 \%$ used condoms and IUDs (intrauterine devices), respectively. Overall, $48.2 \%$ of the participants had laboratory-confirmed sexually transmitted infections, and $19.7 \%$ of the female participants had endogenous infections. After controlling the socio-demographic variables, participants who cleaned their genitals and took a bath less frequently, as well as used condoms or pills had a lower prevalence of sexually transmitted infections, while those who were unwilling to take RTI-related courses and changed their underwear less frequently were more likely to contract sexually transmitted infections. While women who were unwilling to take RTI-related courses had a lower prevalence of endogenous infections, those with more children had a higher prevalence. Conclusion: The status of RTIs among the internal-migrant population of reproductive-age is not optimistic and is related to multiple factors. We believe this study will contribute to improving the knowledge, attitude, and practices related to RTIs.
\end{abstract}


Keywords: reproductive tract infection; knowledge; attitude; and practices (K.A.P); internal migrant population; China

\section{Introduction}

It is estimated that approximately one million new cases of curable sexually transmitted infections (STIs), such as chlamydia, gonorrhea, trichomoniasis, and syphilis, occur daily worldwide in individuals aged 15-49 years [1]. In 2012, the estimated global prevalence of chlamydia, gonorrhea, trichomoniasis, and syphilis among women of reproductive-age was $4.2 \%, 0.8 \%, 5.0 \%$, and $0.5 \%$, respectively, while that among men was $2.7 \%, 0.6 \%, 0.6 \%$, and $0.5 \%$, respectively [1]. In the United States, the prevalence of bacterial vaginosis (BV) among women was $29.2 \%$ [2], and nearly $75 \%$ of adult women have contracted vulvovaginal candidiasis (VVC) at least once in their lifetime (on rare occasions, men can also get genital candidiasis) [3]. In the UK, BV was twice as prevalent as VVC and affected a third of the women at least once in their lifetime [4].

In China, in 2010, the self-reported rate of sexually transmitted diseases (STDs) was 5.4\% and $5.3 \%$ in men and women, respectively [5]. Notably, population mobility was an influencing factor associated with the rising incidence of STDs [6]. In 2015, the internal migrant population of 247 million accounted for $17.9 \%$ of the total Chinese population. The majority $(76.6 \%)$ of these migrants are aged between 15 to 59 years and are characterized by active sexual lives, low educational level, and poor knowledge of reproductive health. Previous studies have reported that $30.3-62.0 \%$ of the internal migrant women population were diagnosed with laboratory-confirmed reproductive tract infections (RTIs), which included BV (11.6-22.2\%), VVC (4.9-28.1\%), trichomoniasis (2.1-12.7\%), gonorrhea $(0.12-1.6 \%)$, syphilis $(0.18-0.2 \%)$, and cervicitis with no laboratory-classification (21.6-59.8\%) [7-11]. However, studies on RTIs among the male internal migrant population are quite rare, though a few of them have reported that $14.9-36.2 \%$ of this population have urogenital tract infections (UTIs) [12,13].

In this study, we aimed to describe the status of RTIs (including STIs) among the reproductive-aged internal-migrant population in China. Meanwhile, knowledge, attitude, and practice (K.A.P) reflects respondents' understanding of any given topic, their feelings and preconceived ideas towards it, and ways in which they demonstrate their knowledge and attitude through their actions [14]. Understanding the levels of K.A.P will enable a more efficient process of awareness creation, as it will allow the program to be tailored more appropriately to the needs of the internal migrants. Thus, we also portrayed RTI-related K.A.P and analyzed the correlation between the K.A.P and RTIs.

\section{Materials and Methods}

\subsection{Definition}

RTIs are caused by bacterial, parasitic, and viral pathogens [15], and include three types of infections: (1) STIs such as chlamydia, gonorrhea, and human immunodeficiency virus (HIV); (2) endogenous infections, such as BV and VVC, that are caused by the overgrowth of organisms normally present in the genital tract of healthy people, and (3) iatrogenic infections, caused by unsafe abortions or poor delivery practices [16]. In this study, the term "RTIs" refers to only STIs and endogenous infections.

\subsection{Sample Size}

The sample size was calculated based on the following formula:

$$
N=\operatorname{deff} \times Z_{\alpha}^{2} \times p \times(1-p) / \delta^{2}
$$


where $N$ is the parameter to be calculated and is the sample size in terms of number of internal migrant population to be selected; deff is the sample design effect, assumed to be 2.0 (default value); $Z_{\alpha}^{2}$ is the statistic that defines the level of confidence desired. The z-statistic to use should be 1.96 for the $95 \%$ confidence interval; $p$ is an estimate of a key indicator to be measured by the study, and here, according to a pilot study carried out in Shanghai with 60 participants, $p$ equals $45 \%$; $\delta$ is the margin of error to be attained. It is recommended to set the level of precision at $10 \%$ of $p$.

Based on the above calculation, a minimal sample size of 940 was required. Therefore, for all the 3 survey sites together, the total sample size was $2820(940 \times 3)$.

\subsection{Sampling Strategy and Study Population}

This study was conducted in the cities of Yinchuan, Urumchi, and Shanghai. In each city, two districts were randomly selected. The sampling cities had to meet the following two criteria. First, the concentration of migrant population was essential, which meant that the selected city (1) possessed intensive industries, concentrated areas in the city and town, and convenient transportation, which are attractive to the internal migrant population; and (2) harbored a sufficiently-large, eligible internal migrant population. Second, the selected areas should have a good foundation for family planning, which indicated: (1) The local government supports the program and pays attention to immigrants' reproductive health; (2) the Department of Service and Management of Migrant Population cooperates with the survey and actively participates in the study; and (3) the selected district is equipped with adequate professional public health agencies (such as community health centers, family planning stations, health education centers, and maternity and child care centers) and medical technical personnel.

Shanghai is the largest city in East China. Yinchuan and Urumchi, as the provincial capitals of Ningxia Hui Autonomous Region and Sinkiang Autonomous Region, respectively, are the main inflowing regions for internal migrants in Northwest China. During the preparation stage, we found that there were only a few studies on the sexual and reproductive health (SRH) of internal migrants in Northwest China. Therefore, Shanghai, Yinchuan, and Urumchi were chosen for this study.

The method of quota-sampling was employed to recruit a composite sample which was approximately proportional to the distribution of internal migrants' occupations: (1) Laborers, who work in manufacturing (textile, plastic products, metal products, and electronic equipment), construction (building, decoration), transport, storage, and expressage; (2) service workers, who have jobs in accommodation, catering, sauna, and entertainment; (3) white collar workers, who are employed in education, accounting, and administrative management; and (4) others, who work in wholesale, retail trades, and so on. The inclusion criteria for eligible respondents were: (1) 15-49 years of age; (2) had resided in the current cities for at least three months; (3) were not registered as permanent residents; and (4) volunteered to participate in this study.

A total of 3771 internal migrants of reproductive age were recruited from March 2016 to February 2017, of which 1174 came from Yinchuan, 1253 from Urumchi, and the remaining 1344 from Shanghai. Of the recruited subjects, 88.0\% (3320/3771; 1124 from Yinchuan, 1015 from Urumchi, and 1181 from Shanghai) participated in the whole investigation, including the questionnaire, clinical examination, and laboratory testing.

\subsection{Measures}

\subsubsection{Socio-Demographic Characteristics}

Conceptual definition: A group of traits related to the size, territorial distribution, and composition of the population, changes therein, and the components of such changes [17]. 
Operational definition: Variables to be assessed are collected from self-administered questionnaires, include area, age, sex, educational level, occupation, marital status, family per capita monthly income (CNY), type of household registration (hukou), duration of staying at the residence per year (Table S1).

\subsubsection{RTI-Related K.A.P}

Conceptual definition: A "knowledge, attitudes, and practices (K.A.P)" survey is a representative study of a specific population that aims to collect data on what is known, believed and done in relation to a particular topic [18]. In this regard, RTI-related K.A.P reflects respondents' understanding of RTIs, their feelings and preconceived ideas about it, and ways in which they demonstrate their knowledge and attitude through their actions [14].

Operational definition: Variables to be assessed are collected from self-administered questionnaires, included knowledge of identification and prevention of RTIs, attitude towards the development of RTI general investigation and willingness to take RTI-related educational courses, practices of personal hygiene (frequency of changing underwear, cleaning one's genitals, and taking a bath, bath methods, and whether or not to have sex during menstruation), whether or not having RTI symptoms in last month, number of sexual partners, number of children (above variable only for the female internal migrant population), and contraceptive methods used (Table S2). The questionnaire for testing knowledge of RTI identification included 21 questions (such as "whether BV is an RTI or not", and "whether frequent micturition, urgent urination, and odynuria are symptoms of RTI or

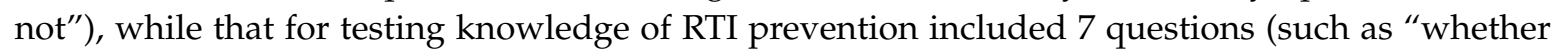
using a condom correctly and consistently is a preventive measure for RTIs or not"). Each question had three alternative answers: "yes", "no", and "do not know", and only one answer was correct. The respondent scored 1 and 0 points for every right and wrong answer, respectively. The sum of the scores from the two tests were then converted to the centesimal system to obtain the final scores for knowledge of RTI identification and prevention, which were used for all further analyses.

\subsubsection{Prevalence of RTIs}

Conceptual definition: The proportion of the investigated population that has laboratory-confirmed STIs or endogenous infections.

Operational definition: Variables to be assessed are collected from laboratory diagnosis results, which included STIs (trichomonal vaginitis (TV), chlamydia trachomatis (CT), neisseria gonorrhoeae (NGH), ureaplasma urealyticum (UU), syphilis, and HIV), as well as endogenous infections (BV and VVC) (Table S3).

Laboratory examination items and diagnostic methods:

Fasting blood, vaginal and cervical discharge (for female) and urethral discharge (for male) were collected.

(1) Trichomoniasis vaginitis

Typical motile trichomonads were identified in a saline wet mount of female vaginal discharge or male urethral discharge.

(2) Chlamydia trachomatis

Nucleic acid amplification test (NAAT) for female endometrial swabs or male urethral swabs: polymerase chain reaction (PCR) was selected in this study.

(3) Neisseria gonorrhoeae

NAAT for female endometrial swabs or male urethral swabs: PCR was selected in this study. 
(4) Ureaplasma urealyticum

NAAT for female endometrial swabs or male urethral swabs: PCR was selected in this study.

(5) Human immunodeficiency virus (HIV)

If an enzyme linked immunosorbent assay (ELSA) test for HIV antibody was positive, a western blotting (WB) test was selected in this study.

(6) Syphilis

Serologic detection of syphilis:

A. If a serologic test of non-treponema pallidum antigen was positive, a rapid plasma reaction actor (RPR) was selected in this study;

B. If a serologic test of non-treponema pallidum antigen was positive, a passive particle agglutination test for treponema pallidum (TPPA) was selected.

(7) Bacterial vaginosis

Meet at least three of the four Amsel diagnostic criteria:

A. Homogeneous white to off-white sticky discharge;

B. Elevated vaginal $\mathrm{pH}(>4.5)$;

C. Fishy odor of vaginal discharge after addition of $1-2$ drops of $10 \%$ potassium hydroxide $(\mathrm{KOH})$;

D. The presence of clue cells in saline wet mount or gram stain of vaginal discharge.

(8) Vulvovaginal candidiasis

Microscopic identification of yeast cells or hyphae in $10 \% \mathrm{KOH}$ in wet-mount preparations of vaginal discharge.

\subsection{Age Standardization}

In consideration of the different age structures in the three cities, it is necessary to standardize the prevalence of RTIs before comparison. Therefore, data on the internal migrant population from the Sixth Nationwide Population Census was selected as the reference population (Table S4).

\section{Ethics Statement}

The study protocol was approved by the Research Ethics Committee of the Shanghai Institute of Planned Parenthood Research (code: PJ2014-20). Before data collection, the purpose and procedures of the study were explained to all the eligible participants. Verbal and written informed consents for privacy protection and information security were obtained from all the participants, including parents of the minors. They fully comprehended their privilege to quit the research at any time they wanted without any adverse consequences.

\section{Results}

\subsection{Socio-Demographics Characteristics.}

In total, 3320 eligible participants (men: $40.7 \%$ and women: 59.3\%) were included in this study. Of the included subjects, 50\% were between the ages of 25-34 years and more than $90 \%$ of them were married. While $76.4 \%$ came from rural areas, $76.9 \%$ had resided in the current residence for a whole year. The majority (42.4\%) of the respondents finished junior high school, and approximately $50 \%$ of the families earned 3000-4999 (CNY) per capita monthly in the last year. Additionally, while 39.9\% of the participants were laborers, $22.6 \%$ were service workers (sauna: $221 / 749$ and entertainment: 241/749) (Table 1). 
Table 1. Socio-demographic characteristics of the study participants.

\begin{tabular}{|c|c|c|c|c|c|c|c|c|c|}
\hline \multirow[t]{2}{*}{ Variables } & \multicolumn{2}{|c|}{$\begin{array}{l}\text { Yinchuan } \\
(N=1124)\end{array}$} & \multicolumn{2}{|c|}{$\begin{array}{c}\text { Urumchi } \\
(N=1015)\end{array}$} & \multicolumn{2}{|c|}{$\begin{array}{l}\text { Shanghai } \\
(N=1181)\end{array}$} & \multicolumn{2}{|c|}{$\begin{array}{c}\text { Total } \\
(N=3320)\end{array}$} & \multirow[t]{2}{*}{$p$-Value ${ }^{\mathrm{b}}$} \\
\hline & $n$ & $\%$ & $n$ & $\%$ & $n$ & $\%$ & $n$ & $\%$ & \\
\hline \multicolumn{9}{|c|}{ Age (years) } & $<0.0001$ \\
\hline $15-24$ & 170 & 15.1 & 105 & 10.3 & 49 & 4.2 & 324 & 9.8 & \\
\hline $25-34$ & 547 & 48.7 & 684 & 67.4 & 431 & 36.5 & 1662 & 50.1 & \\
\hline $35-44$ & 302 & 26.9 & 214 & 21.1 & 435 & 36.8 & 951 & 28.6 & \\
\hline $45-49$ & 105 & 9.3 & 12 & 1.2 & 266 & 22.5 & 383 & 11.5 & \\
\hline \multicolumn{9}{|c|}{ Sex } & $<0.0001$ \\
\hline Male & 372 & 33.1 & 393 & 38.7 & 586 & 49.6 & 1351 & 40.7 & \\
\hline Female & 752 & 66.9 & 622 & 61.3 & 595 & 50.4 & 1969 & 59.3 & \\
\hline \multicolumn{10}{|c|}{ Educational level } \\
\hline Elementary school or lower & 186 & 16.6 & 64 & 6.3 & 124 & 10.5 & 374 & 11.3 & $<0.0001$ \\
\hline Junior high school & 505 & 44.9 & 318 & 31.3 & 584 & 49.5 & 1407 & 42.4 & \\
\hline High school & 265 & 23.6 & 309 & 30.4 & 262 & 22.2 & 836 & 25.2 & \\
\hline College or higher & 168 & 15.0 & 324 & 31.9 & 211 & 17.9 & 703 & 21.2 & \\
\hline \multicolumn{9}{|c|}{ Occupation } & $<0.0001$ \\
\hline Laborer & 295 & 26.3 & 232 & 22.9 & 796 & 67.4 & 1323 & 39.9 & \\
\hline Service worker ${ }^{a}$ & 336 & 29.9 & 341 & 33.6 & 72 & 6.1 & 749 & 22.6 & \\
\hline White-collar worker & 107 & 9.5 & 44 & 4.3 & 191 & 16.2 & 342 & 10.3 & \\
\hline Other & 386 & 34.3 & 398 & 39.2 & 122 & 10.3 & 906 & 27.3 & \\
\hline \multicolumn{9}{|c|}{ Marital status } & $<0.0001$ \\
\hline Married & 1105 & 98.3 & 1014 & 99.9 & 1061 & 89.8 & 3180 & 95.8 & \\
\hline Unmarried & 19 & 1.7 & 1 & 0.1 & 120 & 10.2 & 140 & 4.2 & \\
\hline \multicolumn{9}{|c|}{ Family per capita monthly income (yuan) } & $<0.0001$ \\
\hline$<3000$ & 560 & 49.8 & 144 & 14.2 & 89 & 7.5 & 793 & 23.9 & \\
\hline $3000-4999$ & 394 & 35.1 & 463 & 45.6 & 624 & 52.8 & 1481 & 44.6 & \\
\hline $5000-6999$ & 124 & 11.0 & 274 & 27.0 & 282 & 23.9 & 680 & 20.5 & \\
\hline 7000 and above & 46 & 4.1 & 134 & 13.2 & 186 & 15.8 & 366 & 11.0 & \\
\hline \multicolumn{9}{|c|}{ Type of household registration (hukou) } & $<0.0001$ \\
\hline Rural & 785 & 69.8 & 761 & 75.0 & 991 & 83.9 & 2537 & 76.4 & \\
\hline Urban & 339 & 30.2 & 254 & 25.0 & 190 & 16.1 & 783 & 23.6 & \\
\hline \multicolumn{9}{|c|}{ Duration of staying in current residence per year (month) } & $<0.0001$ \\
\hline$<6$ & 98 & 8.7 & 14 & 34.0 & 34 & 2.9 & 146 & 4.4 & \\
\hline $6 \sim 12$ & 62 & 5.5 & 13 & 547.0 & 547 & 46.3 & 622 & 18.7 & \\
\hline$>12$ & 964 & 85.8 & 988 & 600.0 & 600 & 50.8 & 2552 & 76.9 & \\
\hline
\end{tabular}

a accommodation: 150/749, catering: 137/749, sauna: 221/749; entertainment: $241 / 749 .{ }^{\mathrm{b}}$ Indicates the application of the Cochran-Mantel-Haenszel (CMH) Chi-square test.

\subsection{RTI-Related K.A.P}

\subsubsection{RTI-Related Knowledge}

Of all the participants, $3.2 \%$ and $22.6 \%$ scored 0 points on the RTI identification and RTI prevention knowledge questionnaires, respectively. The median scores for men were 57.1 on both the tests, while for women they were 61.9 (RTI identification) and 57.1 (RTI prevention). Based on the Wilcoxon test, the median score for the knowledge of RTI identification was higher than that for RTI prevention $(S=472,819, p<0.0001)$. While women had a higher median score on the RTI identification knowledge $(\mathrm{Z}=-3.1, p=0.0021)$, men had a higher median score on the RTI prevention knowledge $(\mathrm{Z}=3.7$, $p=0.0002)$ questionnaires.

\subsubsection{RTI-Related Attitude and Practices}

As for RTI associated attitude, while $84.5 \%$ of the participants considered it essential to develop RTI surveillance, 3.1\% thought it was unessential, and the remaining $12.4 \%$ were uncertain. The majority $(96.1 \%)$ of the respondents were willing to take RTI-related educational courses while the remaining 3.9\% were unwilling to. Moreover, explanation/counselling from doctors (63.2\%), brochures $(59.4 \%)$, and lectures $(39.3 \%)$ were the top three preferred education methods (Table 2). 
Table 2. RTI (reproductive tract infections)-related attitude and practices of the study participants.

\begin{tabular}{|c|c|c|c|c|c|c|c|c|}
\hline \multirow{2}{*}{ Variables } & \multicolumn{2}{|c|}{ Yinchuan $(N=1124)$} & \multicolumn{2}{|c|}{ Urumchi $(N=1015)$} & \multicolumn{2}{|c|}{ Shanghai $(N=1181)$} & \multicolumn{2}{|c|}{ Total $(N=3320)$} \\
\hline & $n$ & $\%$ & $n$ & $\%$ & $n$ & $\%$ & $n$ & $\%$ \\
\hline \multicolumn{9}{|l|}{ Attitude } \\
\hline \multicolumn{9}{|c|}{ Whether or not essential to develop RTIs census } \\
\hline Yes & 1058 & 94.1 & 859 & 84.6 & 887 & 75.1 & 2804 & 84.5 \\
\hline No & 53 & 4.7 & 3 & 0.3 & 48 & 4.1 & 104 & 3.1 \\
\hline Uncertain & 13 & 1.2 & 153 & 15.1 & 246 & 20.8 & 412 & 12.4 \\
\hline \multicolumn{9}{|c|}{ Whether or not willing to take RTI-related education courses } \\
\hline Yes & 1106 & 98.4 & 1011 & 99.6 & 1073 & 90.9 & 3190 & 96.1 \\
\hline No & 18 & 1.6 & 4 & 0.4 & 108 & 9.1 & 130 & 3.9 \\
\hline \multicolumn{9}{|c|}{ Preferred education methods ${ }^{a}$} \\
\hline Explanation/counselling from doctors & 884 & 78.6 & 498 & 49.1 & 635 & 53.8 & 2017 & 63.2 \\
\hline Brochures & 921 & 81.9 & 538 & 53.0 & 435 & 36.8 & 1894 & 59.4 \\
\hline Lectures & 865 & 77.0 & 218 & 21.5 & 170 & 14.4 & 1253 & 39.3 \\
\hline Books & 843 & 75.0 & 208 & 20.5 & 137 & 11.6 & 1188 & 37.2 \\
\hline Newspapers & 713 & 63.4 & 145 & 14.3 & 127 & 10.8 & 985 & 30.9 \\
\hline Broadcast/Television & 470 & 41.8 & 332 & 32.7 & 189 & 16.0 & 991 & 31.1 \\
\hline Telephone Hotline & 310 & 27.6 & 256 & 25.2 & 109 & 9.2 & 675 & 21.2 \\
\hline Posters & 343 & 30.5 & 221 & 21.8 & 201 & 17.0 & 765 & 24.0 \\
\hline Others & 110 & 9.8 & 48 & 4.7 & 79 & 6.7 & 237 & 7.4 \\
\hline \multicolumn{9}{|l|}{ Practice } \\
\hline \multicolumn{9}{|c|}{ Personal hygiene } \\
\hline & & dency of & anging & wear & & & & \\
\hline Everyday & 129 & 11.5 & 52 & 5.1 & 994 & 84.2 & 1175 & 35.4 \\
\hline Once in 2-3 days & 785 & 69.8 & 556 & 54.8 & 156 & 13.2 & 1497 & 45.1 \\
\hline Once in $\geq 4$ days & 210 & 18.7 & 407 & 40.1 & 31 & 2.6 & 648 & 19.5 \\
\hline & & quency & cleaning & als & & & & \\
\hline Everyday & 196 & 17.4 & 401 & 39.5 & 1029 & 87.1 & 1626 & 49.0 \\
\hline Once in 2-3 days & 684 & 60.9 & 476 & 46.9 & 129 & 10.9 & 1289 & 38.8 \\
\hline Once in $\geq 4$ days & 244 & 21.7 & 138 & 13.6 & 23 & 2.0 & 405 & 12.2 \\
\hline & & Frequen & of takin & & & & & \\
\hline Everyday & 133 & 11.8 & 83 & 8.2 & 944 & 79.9 & 1160 & 34.9 \\
\hline Once in $2-3$ days & 590 & 52.5 & 329 & 32.4 & 206 & 17.4 & 1125 & 33.9 \\
\hline Once in $\geq 4$ days & 401 & 35.7 & 603 & 59.4 & 31 & 2.6 & 1035 & 31.2 \\
\hline Bath methods & & & & & & & & \\
\hline Shower & 1013 & 90.1 & 987 & 97.2 & 1001 & 84.8 & 3001 & 90.4 \\
\hline Tub bath & 57 & 5.1 & 26 & 2.6 & 116 & 9.8 & 199 & 6.0 \\
\hline Other & 54 & 4.8 & 2 & 0.2 & 64 & 5.4 & 120 & 3.6 \\
\hline & nether or & ving sex & 1 behav & ring me & truation & & & \\
\hline Yes & 287 & 25.5 & 190 & 18.7 & 182 & 15.4 & 659 & 19.8 \\
\hline No & 837 & 74.5 & 825 & 81.3 & 999 & 84.6 & 2661 & 80.2 \\
\hline & Whether & having & I sympt & the las & honth & & & \\
\hline Yes & 313 & 27.9 & 233 & 23.0 & 280 & 23.7 & 826 & 24.9 \\
\hline No & 811 & 72.2 & 782 & 77.0 & 901 & 76.3 & 2494 & 72.1 \\
\hline & & RTI & mptoms & & & & & \\
\hline Excessive or smelly genital secretion & 260 & 23.1 & 232 & 22.9 & 198 & 16.8 & 690 & 83.5 \\
\hline Genital itching & 148 & 13.2 & 148 & 14.6 & 154 & 13.0 & 450 & 54.5 \\
\hline Genital vesicle & 28 & 2.5 & 1 & 0.1 & 87 & 7.4 & 116 & 14.0 \\
\hline Genital excrescence & 30 & 2.7 & 2 & 0.2 & 86 & 7.3 & 118 & 14.3 \\
\hline Pain on urination & 75 & 6.7 & 27 & 2.7 & 123 & 10.4 & 225 & 27.2 \\
\hline & & ntracept & e metho & & & & & \\
\hline No & 210 & 18.7 & 465 & 45.8 & 255 & 21.6 & 930 & 28.0 \\
\hline IUD (intrauterine devices) & 236 & 21.0 & 206 & 20.3 & 267 & 22.6 & 709 & 21.4 \\
\hline Condom & 476 & 42.4 & 252 & 24.8 & 406 & 34.4 & 1134 & 34.2 \\
\hline Pill & 32 & 2.9 & 8 & 0.8 & 27 & 2.3 & 67 & 2.0 \\
\hline Sterilization & 80 & 7.1 & 5 & 0.5 & 158 & 13.4 & 243 & 7.3 \\
\hline Others & 90 & 8.0 & 79 & 7.8 & 68 & 5.8 & 237 & 7.1 \\
\hline & & Jumber & sexual $p$ & & & & & \\
\hline Refusal & 0 & 0.3 & 1 & 0.1 & 30 & 2.5 & 34 & 1.0 \\
\hline 1 & 1116 & 99.3 & 1010 & 99.5 & 1113 & 94.2 & 3239 & 97.6 \\
\hline 2 & 2 & 0.2 & 4 & 0.4 & 27 & 2.3 & 33 & 0.4 \\
\hline$\geq 3$ & 3 & 0.3 & 0 & 0.0 & 11 & 0.9 & 14 & 1.0 \\
\hline & & Numb & of child & & & & & \\
\hline 0 & 173 & 23.0 & 328 & 52.7 & 83 & 14.0 & 584 & 29.7 \\
\hline 1 & 348 & 46.3 & 222 & 35.7 & 201 & 33.8 & 771 & 39.2 \\
\hline 2 & 207 & 27.5 & 57 & 9.2 & 256 & 43.0 & 520 & 26.4 \\
\hline$\geq 3$ & 24 & 3.2 & 15 & 2.4 & 55 & 9.2 & 94 & 4.8 \\
\hline
\end{tabular}

${ }^{a}$ It is a multiple-choice question, and only participants with willingness to take RTI-related education courses answered it. ${ }^{\mathrm{b}}$ It is a multiple-choice question, and only participants with RTI symptoms in the last month answered it. ${ }^{\mathrm{c}}$ Only female participants answered it. 
In terms of RTI-associated practices, while $45.1 \%$ of the participants changed their underwear every 2 or 3 days, $49.0 \%$ cleaned their genitals daily, $34.9 \%$ of them took a bath daily, and the majority of them $(90.4 \%)$ preferred to shower. Meanwhile, approximately $80.2 \%$ of the participants denied having sex during menstruation and $24.9 \%$ reported at least one RTI symptom in the last month. While $28.0 \%$ of the participants did not adopt any contraceptive methods, $47.4 \%$ and $29.7 \%$ of the remaining used condoms and IUDs, respectively. Besides, $97.6 \%$ of the respondents had one sexual partner, $1.0 \%$ had two, $0.4 \%$ had more than three, and $1.0 \%$ denied to answer. While $39.2 \%$ of the women had one child, $29.7 \%$ of them had none (Table 2 ).

Besides, we found the distributions of RTI-related attitude and practices (whether or not willing to take RTI-related education courses, frequency of changing underwear, frequency of cleaning genitals, frequency of taking bath, bath methods, whether or not having RTI symptoms in the last month, contraceptive methods in use, number of sexual partners and number of children) were different in each age group $(p<0.05)$. Younger participants were more inclined to take RTI-related education courses and preferred to shower. Participants in the older age group; however, more frequently changed underwear, cleaned genitals, and took a bath. More of the older group had RTI-symptoms in the last month, used contraceptives, and had more sexual partners. They mostly adopted IUD and sterilization rather than condoms, which was more popular among the younger. In addition, older female participants had more children.

\subsection{Laboratory-Confirmed RTIs}

Overall, $48.2 \%$ of the study participants had laboratory-confirmed STIs, and $19.7 \%$ of the women had endogenous infections. Among those with STIs, 44.0\% were men, and 51.0\% were women. Moreover, the rate of UU infection, which was the most common, was $41.3 \%$ in men and $48.3 \%$ in women. Table 3 summarizes the information on laboratory-confirmed RTIs among participants in each city. Using the internal migrant population from the Sixth Nationwide Population Census as the reference population, the total age-adjusted rate of STIs was $47.8 \%$, and that of endogenous infections was $17.6 \%$. The age-adjusted rates of STIs in Yinchuan, Urumchi, and Shanghai were $40.9 \%, 46.2 \%$, and $57.6 \%$, respectively, that among men were $38.7 \%, 40.8 \%$, and $49.3 \%$, respectively, and those in women were $41.8 \%, 47.7 \%$, and $71.0 \%$, respectively. The age-adjusted rates of endogenous infections in Yinchuan, Urumchi, and Shanghai were $18.8 \%, 21.9 \%$, and $11.4 \%$, respectively (Table 4 ).

Table 3. RTI-related attitude and practices of the study participants in four age groups

\begin{tabular}{|c|c|c|c|c|c|c|c|c|c|}
\hline \multirow[t]{2}{*}{ Variables } & \multicolumn{2}{|c|}{$\begin{array}{c}15-24 \text { years old } \\
(N=324)\end{array}$} & \multicolumn{2}{|c|}{$\begin{array}{c}25-34 \text { years old } \\
(N=1662)\end{array}$} & \multicolumn{2}{|c|}{$\begin{array}{c}35-44 \text { years old } \\
(N=951)\end{array}$} & \multicolumn{2}{|c|}{$\begin{array}{c}45-49 \text { years old } \\
(N=383)\end{array}$} & \multirow[t]{2}{*}{$p$-Value ${ }^{c}$} \\
\hline & $n$ & $\%$ & $n$ & $\%$ & $n$ & $\%$ & $n$ & $\%$ & \\
\hline \multicolumn{10}{|l|}{ Attitude } \\
\hline \multicolumn{9}{|c|}{ Whether or not essential to develop RTIs census } & 0.8586 \\
\hline Yes & 262 & 80.9 & 1419 & 85.4 & 806 & 84.8 & 317 & 82.8 & \\
\hline No & 17 & 5.2 & 47 & 2.8 & 28 & 2.9 & 12 & 3.1 & \\
\hline Uncertain & 45 & 13.9 & 196 & 11.8 & 117 & 12.3 & 54 & 14.1 & \\
\hline \multicolumn{9}{|c|}{ Whether or not willing to take RTI-related education courses } & $<0.0001$ \\
\hline Yes & 317 & 97.8 & 1624 & 97.7 & 898 & 94.4 & 351 & 91.6 & \\
\hline No & 7 & 2.2 & 38 & 2.3 & 53 & 5.6 & 32 & 8.4 & \\
\hline \multicolumn{10}{|c|}{ Preferred education methods ${ }^{a}$} \\
\hline Explanation/counselling from doctors & 191 & 59.0 & 989 & 59.5 & 601 & 63.2 & 236 & 61.6 & 0.0228 \\
\hline Brochures & 185 & 57.1 & 987 & 59.4 & 537 & 56.5 & 185 & 48.3 & 0.0122 \\
\hline Lectures & 135 & 41.7 & 685 & 41.2 & 313 & 32.9 & 120 & 31.3 & $<0.0001$ \\
\hline Books & 140 & 43.2 & 631 & 38.0 & 322 & 33.9 & 95 & 24.8 & $<0.0001$ \\
\hline Newspapers & 107 & 33.0 & 493 & 29.7 & 280 & 29.4 & 105 & 27.4 & 0.4559 \\
\hline Broadcast / Television & 124 & 38.3 & 524 & 31.5 & 270 & 28.4 & 73 & 19.1 & $<0.0001$ \\
\hline Telephone Hotline & 62 & 19.1 & 384 & 23.1 & 176 & 18.5 & 53 & 13.8 & 0.0037 \\
\hline Posters & 55 & 17.0 & 390 & 23.5 & 244 & 25.7 & 76 & 19.8 & 0.0189 \\
\hline Others & 16 & 4.9 & 105 & 6.3 & 78 & 8.2 & 38 & 9.9 & 0.0189 \\
\hline
\end{tabular}


Table 3. Cont

\begin{tabular}{|c|c|c|c|c|c|c|c|c|c|}
\hline \multirow[t]{2}{*}{ Variables } & \multicolumn{2}{|c|}{$\begin{array}{c}15-24 \text { years old } \\
(N=324)\end{array}$} & \multicolumn{2}{|c|}{$\begin{array}{c}25-34 \text { years old } \\
(N=1662)\end{array}$} & \multicolumn{2}{|c|}{$\begin{array}{c}\text { 35-44 years old } \\
(N=951)\end{array}$} & \multicolumn{2}{|c|}{$\begin{array}{c}45-49 \text { years old } \\
(N=383)\end{array}$} & \multirow[t]{2}{*}{$p$-Value $^{c}$} \\
\hline & $n$ & $\%$ & $n$ & $\%$ & $n$ & $\%$ & $n$ & $\%$ & \\
\hline \multicolumn{10}{|l|}{ Practice } \\
\hline \multicolumn{10}{|c|}{ Personal hygiene } \\
\hline \multicolumn{9}{|c|}{ Frequency of changing underwear } & $<0.0001$ \\
\hline Everyday & 57 & 17.6 & 435 & 26.2 & 442 & 46.5 & 241 & 62.9 & \\
\hline Once in $2-3$ days & 180 & 55.6 & 862 & 51.9 & 360 & 37.9 & 95 & 24.8 & \\
\hline Once in $\geq 4$ days & 87 & 26.9 & 365 & 21.9 & 149 & 15.7 & 47 & 12.3 & \\
\hline \multicolumn{9}{|c|}{ Frequency of cleaning genitals } & $<0.0001$ \\
\hline Everyday & 106 & 32.7 & $721^{\circ}$ & 43.4 & 540 & 56.8 & 259 & 67.6 & \\
\hline Once in $2-3$ days & 165 & 50.9 & 716 & 43.1 & 324 & 34.1 & 84 & 21.9 & \\
\hline Once in $\geq 4$ days & 53 & 16.4 & 225 & 13.5 & 87 & 9.2 & 40 & 10.4 & \\
\hline \multicolumn{9}{|c|}{ Frequency of taking bath } & $<0.0001$ \\
\hline Everyday & 77 & 23.8 & 450 & 27.1 & 407 & 42.8 & 226 & 59.0 & \\
\hline Once in $2-3$ days & 132 & 40.7 & 618 & 37.2 & 276 & 29.0 & 99 & 25.9 & \\
\hline Once in $\geq 4$ days & 115 & 35.5 & 594 & 35.7 & 268 & 28.2 & 58 & 15.1 & \\
\hline \multicolumn{9}{|c|}{ Bath methods } & $<0.0001$ \\
\hline Shower & 288 & 88.9 & 1564 & 94.1 & 830 & 87.3 & 319 & 83.3 & \\
\hline Tub bath & 16 & 4.9 & 71 & 4.3 & 68 & 7.2 & 44 & 11.5 & \\
\hline Other & 20 & 6.2 & 27 & 1.6 & 53 & 5.6 & 20 & 5.2 & \\
\hline \multicolumn{9}{|c|}{ Whether or not having sexual behavior during menstruation } & 0.8108 \\
\hline Yes & 79 & 24.4 & 312 & 18.8 & 185 & 19.5 & 83 & 21.7 & \\
\hline No & 245 & 75.6 & 1350 & 81.2 & 766 & 80.6 & 300 & 78.3 & \\
\hline \multicolumn{9}{|c|}{ Whether or not having RTI symptoms in the last month } & 0.0421 \\
\hline Yes & 64 & 19.8 & 408 & 24.6 & 256 & 26.9 & 98 & 25.6 & \\
\hline No & 260 & 80.3 & 1254 & 75.4 & 695 & 73.1 & 285 & 74.4 & \\
\hline \multicolumn{9}{|c|}{ Contraceptive methods in use } & $<0.0001$ \\
\hline No & 140 & 43.2 & 504 & 30.3 & 198 & 20.8 & 88 & 23.0 & \\
\hline IUD & 37 & 11.4 & 297 & 17.9 & 279 & 29.3 & 96 & 25.1 & \\
\hline Condom & 117 & 36.1 & 654 & 39.4 & 300 & 31.6 & 63 & 16.5 & \\
\hline Pill & 5 & 1.5 & 29 & 1.7 & 25 & 2.6 & 8 & 2.1 & \\
\hline Sterilization & 2 & 0.6 & 50 & 3.0 & 99 & 10.4 & 92 & 24.0 & \\
\hline Others & 23 & 7.1 & 128 & 7.7 & 50 & 5.3 & 36 & 9.4 & \\
\hline \multicolumn{9}{|c|}{ Number of sexual partners } & 0.0171 \\
\hline Refusal & 2 & 0.9 & 4 & 0.6 & 12 & 1.3 & 9 & 2.4 & \\
\hline 1 & 316 & 97.5 & 1614 & 98.7 & 923 & 97.1 & 359 & 93.7 & \\
\hline 2 & 3 & 0.9 & 9 & 0.5 & 6 & 1.4 & 8 & 2.1 & \\
\hline$\geq 3$ & 2 & 0.6 & 2 & 0.1 & 3 & 0.3 & 7 & 1.8 & \\
\hline \multicolumn{9}{|c|}{ Number of children ${ }^{b}$} & 0.0029 \\
\hline 0 & 130 & 61.0 & 348 & 35.2 & 80 & 14.2 & 26 & 12.8 & \\
\hline 1 & 66 & 31.0 & 433 & 43.8 & 219 & 38.8 & 53 & 26.1 & \\
\hline 2 & 17 & 8.0 & 189 & 19.1 & 219 & 38.8 & 95 & 46.8 & \\
\hline$\geq 3$ & 0 & 0.0 & 18 & 1.8 & 47 & 8.3 & 29 & 14.3 & \\
\hline
\end{tabular}

${ }^{a}$ It is a multiple-choice question, and only participants with willingness to take RTI-related education courses answered it. ${ }^{b}$ Only female participants answered it. ${ }^{c}$ Indicates the application of the CMH Chi-square test. 
Table 4. Distribution of laboratory-confirmed RTIs among three cities.

\begin{tabular}{|c|c|c|c|c|c|c|c|c|c|c|c|c|}
\hline \multirow[b]{2}{*}{ Variables } & \multicolumn{4}{|c|}{ Yinchuan } & \multicolumn{4}{|c|}{ Urumchi } & \multicolumn{4}{|c|}{ Shanghai } \\
\hline & $\begin{array}{c}\text { Male } \\
(\%)\end{array}$ & $\begin{array}{c}\text { Female } \\
(\%)\end{array}$ & $p$-Value & $\begin{array}{c}\text { Total } \\
(\%)\end{array}$ & $\begin{array}{c}\text { Male } \\
(\%)\end{array}$ & $\begin{array}{c}\text { Female } \\
(\%)\end{array}$ & $p$-Value & $\begin{array}{c}\text { Total } \\
(\%)\end{array}$ & $\begin{array}{c}\text { Male } \\
(\%)\end{array}$ & $\begin{array}{c}\text { Female } \\
(\%)\end{array}$ & $p$-Value & $\begin{array}{c}\text { Total } \\
\text { (\%) }\end{array}$ \\
\hline Bacterial vaginosis (BV) & - & 16.4 & - & - & - & 17.4 & - & - & - & 15.0 & - & - \\
\hline Vulvovaginal candidiasis (VVC) & - & 5.3 & - & - & - & 3.7 & - & - & - & 3.9 & - & - \\
\hline Endogenous infections & - & 20.4 & - & 20.4 & - & 21.1 & - & 21.0 & - & 17.5 & - & 19.7 \\
\hline Trichomonal vaginitis (TV) & - & 0.1 & - & - & - & 3.1 & - & - & - & 1.2 & - & - \\
\hline Chlamydia trachomatis (CT) & 4.3 & 4.5 & 0.8663 & 4.5 & 5.3 & 5.5 & 0.9330 & 5.4 & 7.0 & 7.7 & 0.6292 & 7.4 \\
\hline Neisseria gonorrhoeae (NGH) & 0.0 & 0.3 & 0.3197 & 0.2 & 0.0 & 0.2 & 0.4267 & 0.1 & 0.2 & 0.2 & 0.9914 & 0.2 \\
\hline Ureaplasma urealyticum (UU) & 39.3 & 37.0 & 0.4584 & 37.7 & 38.4 & 48.9 & 0.0011 & 44.8 & 44.5 & 62.2 & $<0.0001$ & 53.4 \\
\hline Human immunodeficiency virus (HIV) & 0.0 & 0.0 & - & 0.0 & 0.0 & 0.0 & - & 0.0 & 0.2 & 0.0 & - & 0.1 \\
\hline Syphilis & 0.5 & 0.4 & 0.7424 & 0.4 & 2.8 & 0.8 & 0.0130 & 1.6 & 0.9 & 0.2 & 0.0979 & 0.5 \\
\hline Sexually transmitted infections (STIs) & 39.8 & 39.9 & 0.9721 & 39.9 & 39.7 & 50.5 & 0.0008 & 46.3 & 49.7 & 65.7 & $<0.0001$ & 48.2 \\
\hline
\end{tabular}




\subsection{Univariable and Multivariable Analyses of RTIs}

In the univariable analyses of RTIs, the following factors were associated with both STIs and endogenous infections: age, education attainment, occupation, belief in the necessity to develop RTI surveillance, willingness to take RTI-related education, whether or not having RTI symptoms in the last month, and contraceptive methods.

For STIs, other associated factors included region, sex, family per capita monthly income, type of household registration (hukou), the frequency of cleaning genitals, and frequency of taking a bath. Factors that were associated with endogenous infections included bath methods and the number of children (Table 5).

Table 5. Univariable analyses of the correlation between socio-demographic characteristics and RTIs among the internal migrant population.

\begin{tabular}{|c|c|c|c|c|c|c|}
\hline \multirow{2}{*}{ Variables } & \multicolumn{3}{|c|}{ Sexually Transmitted Infections } & \multicolumn{3}{|c|}{ Endogenous Infections (Women) } \\
\hline & $n$ & $\%$ & $p$-Value ${ }^{a}$ & $n$ & $\%$ & $p$-Value ${ }^{\text {a }}$ \\
\hline \multicolumn{7}{|c|}{ Socio-demographic characteristics } \\
\hline \multicolumn{7}{|c|}{ Area } \\
\hline Yinchuan & 448 & 39.9 & \multirow{3}{*}{$<0.0001$} & 153 & 13.6 & \multirow{3}{*}{0.2491} \\
\hline Urumchi & 470 & 46.3 & & 131 & 12.9 & \\
\hline Shanghai & 682 & 57.8 & & 104 & 8.8 & \\
\hline \multicolumn{7}{|c|}{ Age $^{b}$} \\
\hline $15-24$ & 147 & 45.4 & \multirow{4}{*}{$<0.0001$} & 24 & 7.4 & \multirow{4}{*}{$<0.0001$} \\
\hline $25-34$ & 733 & 44.1 & & 191 & 11.5 & \\
\hline $35-44$ & 483 & 50.8 & & 124 & 13.0 & \\
\hline $45-49$ & 237 & 61.9 & & 49 & 12.8 & \\
\hline \multicolumn{7}{|c|}{ Sex ${ }^{b}$} \\
\hline Male & 595 & 44.0 & \multirow{2}{*}{$<0.0001$} & - & - & \multirow[t]{2}{*}{ - } \\
\hline Female & 1005 & 51.0 & & - & - & \\
\hline \multicolumn{7}{|c|}{ Educational level ${ }^{\mathrm{b}}$} \\
\hline Elementary school or lower & 199 & 53.2 & \multirow{4}{*}{$<0.0001$} & 76 & 20.3 & \multirow{4}{*}{0.0064} \\
\hline Junior high school & 711 & 50.5 & & 167 & 11.9 & \\
\hline High school & 404 & 48.3 & & 91 & 10.9 & \\
\hline College or higher & 286 & 40.7 & & 54 & 7.7 & \\
\hline \multicolumn{7}{|c|}{ Occupation ${ }^{b}$} \\
\hline Laborer & 721 & 54.5 & \multirow{4}{*}{0.0006} & 114 & 8.6 & \multirow{4}{*}{$<0.0001$} \\
\hline Service worker & 325 & 43.4 & & 139 & 18.6 & \\
\hline White-collar worker & 141 & 41.2 & & 21 & 6.1 & \\
\hline Other & 413 & 45.6 & & 114 & 12.6 & \\
\hline \multicolumn{7}{|c|}{ Family per capita monthly income (yuan) ${ }^{b}$} \\
\hline$<3000$ & 330 & 41.6 & \multirow{4}{*}{0.0537} & 124 & 15.6 & \multirow{4}{*}{0.1528} \\
\hline $3000-4999$ & 729 & 49.2 & & 166 & 11.2 & \\
\hline $5000-6999$ & 367 & 54.0 & & 75 & 11.0 & \\
\hline 7000 and above & 174 & 47.5 & & 23 & 6.3 & \\
\hline \multicolumn{7}{|c|}{ Type of household registration (hukou) ${ }^{b}$} \\
\hline Rural & 1270 & 50.1 & \multirow[b]{2}{*}{0.0067} & 298 & 11.8 & \multirow[b]{2}{*}{0.4074} \\
\hline Urban & 330 & 42.2 & & 90 & 11.5 & \\
\hline & related & (knov & e; attitude; & practic & & \\
\hline Whethe & not th & is esse & to develop s & illance & $\mathrm{II}^{\mathrm{b}}$ & \\
\hline Yes & 1303 & 46.5 & & 348 & 12.4 & \\
\hline No & 55 & 52.9 & 0.0168 & 1 & 1.0 & 0.0272 \\
\hline Uncertain & 242 & 58.7 & & 39 & 9.5 & \\
\hline & ther or & illing & RTI-relate & ucatio & & \\
\hline Yes & 1515 & 47.5 & & 381 & 11.9 & \\
\hline No & 85 & 65.4 & 0.0018 & 7 & 5.4 & 0.0256 \\
\hline
\end{tabular}


Table 5. Cont.

\begin{tabular}{|c|c|c|c|c|c|c|}
\hline \multirow{2}{*}{ Variables } & \multicolumn{3}{|c|}{ Sexually Transmitted Infections } & \multicolumn{3}{|c|}{ Endogenous Infections (Women) } \\
\hline & $n$ & $\%$ & $p$-Value ${ }^{a}$ & $n$ & $\%$ & $p$-Value ${ }^{\text {a }}$ \\
\hline \multicolumn{7}{|c|}{ Frequency of cleaning genitals ${ }^{b}$} \\
\hline Everyday & 901 & 55.4 & & 179 & 11.0 & \\
\hline Once in $2-3$ days & 547 & 42.4 & 0.0003 & 168 & 13.0 & 0.4513 \\
\hline Once in $\geq 4$ days & 152 & 37.5 & & 41 & 10.1 & \\
\hline \multicolumn{7}{|c|}{ Frequency of taking bath ${ }^{b}$} \\
\hline Everyday & 669 & 57.7 & & 106 & 9.1 & \\
\hline Once in $2-3$ days & 508 & 45.2 & 0.0004 & 142 & 12.6 & 0.1068 \\
\hline Once in $\geq 4$ days & 423 & 40.9 & & 140 & 13.5 & \\
\hline \multicolumn{7}{|c|}{ Bath methods $b$} \\
\hline Shower & 1431 & 47.7 & & 353 & 11.8 & \\
\hline Tub bath & 113 & 56.8 & 0.2100 & 28 & 14.1 & 0.0951 \\
\hline Other & 56 & 46.7 & & 7 & 5.8 & \\
\hline \multicolumn{7}{|c|}{ Whether or not having RTI symptoms in the last month ${ }^{b}$} \\
\hline Yes & 449 & 54.4 & & 222 & 32.9 & \\
\hline No & 1151 & 46.2 & $<0.0001$ & 166 & 12.8 & $<0.0001$ \\
\hline \multicolumn{7}{|c|}{ Contraceptive methods $b$} \\
\hline None & 472 & 50.8 & & 88 & 9.5 & \\
\hline IUD & 370 & 52.2 & & 94 & 13.3 & \\
\hline Condom & 473 & 41.7 & & 116 & 10.2 & \\
\hline Pills & 24 & 35.8 & $<0.0001$ & 13 & 19.4 & 0.0685 \\
\hline Sterilization & 147 & 60.5 & & 38 & 15.6 & \\
\hline Other & 114 & 48.1 & & 39 & 16.5 & \\
\hline \multicolumn{7}{|c|}{ Number of children ${ }^{b}$} \\
\hline 0 & - & - & & 87 & 9.1 & \\
\hline 1 & - & - & & 145 & 14.0 & \\
\hline 2 & - & - & - & 131 & 21.0 & $<0.0001$ \\
\hline$\geq 3$ & - & - & & 25 & 22.1 & \\
\hline
\end{tabular}

Notation: Marital status, duration of stay in residence (months), the frequency of changing underwear, whether or not having sexual behavior during menstruation, and the number of sexual partners all showed no significant association with STIs and endogenous infections. ${ }^{a}$ Indicates the application of the CMH Chi-square test. ${ }^{\mathrm{b}}$ Implicates the adjusted region effect.

The socio-demographic characteristics and RTI-related K.A.P variables were used for the multivariable analyses of STIs and endogenous infections. Consequently, the occurrence of STIs was notably related with a willingness to take RTI-related educational courses, RTI symptoms, contraceptive methods, and the frequency of changing underwear, cleaning genitals, and taking a bath $(p<0.05)$. If study participants were unwilling to take RTI-related courses, the occurrence of STIs was more likely to increase (OR $=1.6,95 \% \mathrm{CI}$ : 1.1-2.3). Additionally, while STIs were more likely to occur in participants who changed their underwear less frequently, they were less likely to occur in those with a lower frequency of cleaning genitals and taking a bath. While for the study participants who had RTI symptoms in last month, the occurrence of STIs was more likely to increase (OR $=1.3,95 \%$ CI: 1.1-1.6). The study respondents who used condoms or pills for contraception were less likely to have STIs than those who used sterilization (Table 6).

Multivariable analyses also revealed that whether participants were willing to take RTI-related courses or not, RTI symptoms and the number of children were significant associated with endogenous infections $(p<0.05)$. The prevalence of these infections was, however, less likely to increase in participants who were unwilling to take RTI-related courses (OR $=0.4,95 \%$ CI: 0.2-0.9). Additionally, the respondents who had RTI symptoms in last month were more likely to have endogenous infections $(\mathrm{OR}=3.2,95 \% \mathrm{CI}: 2.5-4.0)$. Furthermore, the probability of having endogenous infections was significantly higher in participants who had more than one child (Table 6). 
Table 6. Multivariable analyses of the correlation between RTI-related K.A.P and prevalence of RTIs among the internal migrant population.

\begin{tabular}{|c|c|c|c|c|c|c|c|}
\hline \multicolumn{2}{|c|}{ Variables } & \multicolumn{3}{|c|}{ Sexually Transmitted Infections } & \multicolumn{3}{|c|}{$\begin{array}{l}\text { Endogenous Infections } \\
\text { (Female) }\end{array}$} \\
\hline Comparable G & Reference G & Odds Ratio (OR) & $\begin{array}{l}\text { 95\% Confidence } \\
\text { Interval (CI) }\end{array}$ & $p$-Value & OR & $95 \%$ CI & $p$-Value \\
\hline \multicolumn{8}{|c|}{ RTI-related K.A.P } \\
\hline \multicolumn{8}{|c|}{ Attitude } \\
\hline \multicolumn{8}{|c|}{ Whether or not willing to take RTI-related educational courses } \\
\hline No & Yes & 1.6 & $1.1-2.3$ & 0.0201 & 0.4 & $0.2-0.9$ & 0.0226 \\
\hline \multicolumn{8}{|c|}{ Practices } \\
\hline \multicolumn{8}{|c|}{ Frequency of changing underwear } \\
\hline Once in 2-3 days & & 1.5 & $1.1-1.9$ & 0.0058 & - & - & - \\
\hline Once in $\geq 4$ days & Everyday & 1.8 & $1.3-2.6$ & 0.0013 & - & - & - \\
\hline \multicolumn{8}{|c|}{ Frequency of cleaning genitals } \\
\hline Once in 2-3 days & Fyeryday & 0.8 & $0.6-0.9$ & 0.0136 & - & - & - \\
\hline Once in $\geq 4$ days & Everyday & 0.7 & $0.5-1.0$ & 0.0567 & - & - & - \\
\hline \multicolumn{8}{|c|}{ Frequency of taking a bath } \\
\hline Once in $2-3$ days & & 0.8 & $0.6-1.0$ & 0.0272 & - & - & - \\
\hline Once in $\geq 4$ days & Everyday & 0.5 & $0.4-0.7$ & $<0.0001$ & - & - & - \\
\hline \multicolumn{8}{|c|}{ Whether or not having RTI symptoms in the last month } \\
\hline Yes & No & 1.3 & $1.1-1.6$ & 0.0008 & 3.2 & $2.5-4.0$ & $<0.0001$ \\
\hline \multicolumn{8}{|c|}{ Number of children } \\
\hline 1 & & - & - & - & 1.2 & $0.9-1.7$ & 0.2740 \\
\hline 2 & 0 & - & - & - & 1.7 & $1.1-2.5$ & 0.0112 \\
\hline$\geq 3$ & & - & - & - & 1.8 & $1.0-3.3$ & 0.0624 \\
\hline \multicolumn{8}{|c|}{ Contraceptive methods } \\
\hline None & & 0.9 & $0.6-1.2$ & 0.4621 & - & - & - \\
\hline IUD & & 0.97 & $0.6-1.20$ & 0.3844 & - & - & - \\
\hline Condom & Sterilization & 0.7 & $0.5-0.9$ & 0.0161 & - & - & - \\
\hline Pills & & 0.4 & $0.2-0.8$ & 0.0055 & - & - & - \\
\hline Others & & 0.7 & $0.5-1.1$ & 0.1214 & - & - & - \\
\hline
\end{tabular}

Notation: Socio-demographic characteristics, including area, age, sex, educational level, occupation, marital status, family per capita monthly income (yuan), type of household registration (hukou), and duration of stay in the current residence per year (months), were all controlled during multivariable analyses.

\section{Discussion}

This population-based study reveals poor reproductive health among the young internal migrant population in three Chinese cities. While $44.0 \%$ and $51.0 \%$ of the male and female participants, respectively, had laboratory-confirmed STIs, $19.7 \%$ of the female participants had endogenous infections. To the best of our knowledge, multiple previous studies have emphasized on women SRH, and have shown that the prevalence of RTIs in women varies from $18.5 \%$ to as high as $64.0 \%$ [19-23]. Only a few population-based studies on male SRH have revealed that $6.1-36.2 \%$ of the population are positive for STIs $[12,13,24]$. These variations might be attributed to factors such as different study profiles, and diagnosis criteria (whether it was self-reported or clinical diagnosis based on laboratory examination). A careful speculum and bimanual examination, a genital secretion test, and a blood test for both men and women can help identify multiple silent STIs/RTIs. After adjusting for age, the standardized rate of STIs was highest in Shanghai, which might be due to the population mobility and occupation distribution. Shanghai, is a city with the most mobile population and accounts for $4.3 \%$ of China's internal migrant population. As previous studies have shown, risky sexual behaviors such as multiple partners, casual partners, and no condom use during the most recent sexual intercourse with the regular partner, were all significantly higher among the nonresidents compared to the residents in both men and women [25]. The univariable analysis showed that the highest prevalence of STIs was among laborers, who accounted for the majority of the internal migrants in Shanghai. Shendre et al. have also reported higher chances of developing STDs in unskilled workers [26].

Urumchi had the highest standardized rate of endogenous infections, which is probably due to poor hygienic habits and occupations. In Urumchi, while $95.8 \%$ of the subjects changed their 
underwear once in more than two days, $64.9 \%$ of them cleaned their genitals once in more than every two days, and $15.4 \%$ of them indulged in sexual behavior during menstruation. Moreover, in this study, the majority of Urumchi participants were engaged in service work, which is a risk factor for endogenous infections.

Ureaplasma, one of the most prevalent genotypes of mycoplasma, was the most common causative pathogen in both men and women with UU. Mycoplasma is an opportunistic pathogen that always coexists with host asymptomatically. UU, during pregnancy (16-20 weeks of gestational age), combined with other factors such as BV or cervical incompetence, often leads to premature rupture of membrane (PROM), preterm birth, stillbirth or fetal death, low birth weight, congenital malformations, and mycoplasmal pneumonia especially in preterm neonates $[27,28]$. Besides, UU is also associated with the male reproductive system and is more prevalent in infertile men than in fertile men [29]. An experimental study on rats has indicated that UU affects the sperm morphology leading to its death [30], though other studies have ruled out any association between UU and adverse fertility outcomes [31,32].

Adequate knowledge of RTIs could encourage appropriate prevention measures [33] and decrease the incidence of low genital tract infections [23]. In this study, the overall RTI-related knowledge was quite poor, with $3.2 \%$ and $22.6 \%$ of the participants scoring 0 points on the knowledge of RTI identification and RTI prevention questionnaires, respectively. Health-related decisions and actions of the participants are affected by their attitude towards preventing health problems [34]. Unwillingness to take RTI-related educational courses; therefore, impedes their chance to get appropriate information on reproductive health, including transmission and symptoms of RTIs, and appropriate family planning methods, thereby increasing the likelihood of exposure to these risk factors. In this study, frequent cleaning of one's genitals and bathing have been associated with a high prevalence of STIs. Although, STIs are mostly caused by pathogens via unsafe and unprotected sexual behavior with multiple sexual partners or disloyal partners, many studies have demonstrated that genital hygiene practices also correlate with higher risks of STIs [35-37]. Inappropriate cleaning methods, such as vaginal touching, use of feminine wipes and alkaline lotion for genital and reproductive tract inevitably increase the chances of infection. In our qualitative interviews, we found that the majority of study participants, especially women used antiseptic wipes to clean their genitals, and many of these wipes in the Chinese market are of substandard quality [38]. All these factors disrupt the vaginal ecological balance and induce dysbacteriosis. Correct and consistent use of condoms can greatly reduce the risks of both pregnancy and STIs, including HIV transmission [39]. Condoms form a barrier to prevent direct contact with body fluids [40] and is the only and most effective contraceptive method that helps prevent the spread of STIs [41]. Oral contraceptives present no protection against viral STIs and $\mathrm{HIV}$, and yeast infections are more common in people taking pills [42]. Meanwhile, many previous studies on birth control methods and STIs have revealed that oral or injectable hormonal contraceptives facilitate the risk of acquiring certain STDs, such as chlamydia and vaginal candidiasis, but decrease the risk for bacterial vaginosis [43-45]. However, contrary to the above findings, we found that oral contraceptives have an opposite relationship with STIs and have an insignificant relationship with endogenous infections. This could be due to the inadequate statistical power for the small sample size of oral contraceptive users $(67 / 3320)$. As for endogenous infections, the more children the participant had, the greater were the odds of her contracting these infections. More children indicated more pregnancies and deliveries. Pregnancy is accompanied by compromised immunity and a higher incidence of yeast infections [42]. Endogenous infections are usually associated with hypoimmunity $[27,46]$ and are characterized by repeated episodes. Delivery often causes soft birth canal injury, which in turn leads to infection. Some of our findings are consistent with previous reports $[22,47,48]$. Specifically, significant differences have been seen in the prevalence of STIs and endogenous infections in different socio-demographic contexts. In multivariable analyses, while age and sex were associated with the occurrence of STIs, age, occupation, and family per capita monthly income correlated with endogenous infections. Compared to the older participants, resistance to 
infections and sex hormone levels (which helps in strengthening vaginal immunity) were much better among the younger participants [27]. Meanwhile, women were more vulnerable to infections due to biological and social factors [47,49]. In this study, the majority of service workers were recruited from sauna rooms, entertainment venues, and hotels. These people often had irregular routines, high-intensity jobs, and unhealthy working environments. On the other hand, participants with higher income levels were more eager and able to improve their quality of life, including cleaner surroundings, accessible health services [48], and maintaining physical and mental health.

In light of the differences in the prevalence of STIs and endogenous infections in the three cities and their correlation with K.A.P, local governments need to establish reproductive health policies to emphasize the importance of maintaining good SRH. Simultaneously, explanation/counselling from doctors, brochures, and lectures, which were found to be the most popular education methods, could be adopted by reproductive health programs to improve the utilization of basic public services and cultivate reproductive health consciousness and behaviors. Health education centers and primary medical care units should provide convenient access for diagnosis and treatment of RTIs in workplace and community to facilitate health service seeking behavior.

This was a large multi-centric population-based cross-sectional study of the internal migrant population, which highlights the status of RTIs and related K.A.P. in this population. Moreover, we analyzed the relationship between K.A.P and RTIs. The study is beneficial to the subsequent intervention programs. However, there are some limitations to this study. First, there was a shortage of more common K.A.P. information, like sexual experience, number of homosexual sexual partners, access to and past behaviors of seeking care for RTIs symptoms, which might generate a limited correlation between current RTIs-related K.A.P and RTIs. Second, due to the private and sensitive nature of sexual behavior and the number of sexual partners, its impact on RTIs might have possibly been underestimated. Third, detailed information on the cleaning materials used, especially for the genitals, were collected by qualitative interviews rather than a structured questionnaire. Fourth, this is a cross-sectional study that will inevitably impede the drawing of causal inference.

\section{Conclusions}

This study documents a moderately high prevalence of RTIs among the internal migrant population of reproductive-age in three cities in China. Our results show that the knowledge of RTI identification and prevention among this population is quite poor. Additionally, the majority of respondents believed it was essential to develop RTI surveillance and showed great enthusiasm for SRH education. We have also demonstrated a correlation between K.A.P and the prevalence of RTIs in this population, after adjusting for the socio-demographic variables. To the best of our knowledge, there are only a few studies that have evaluated the prevalence of STIs and endogenous infections in male and female internal migrants, to separately explore the relationship between STIs/endogenous infections and K.A.P in China. This study emphasizes the need for better RTI-related K.A.P to improve the reproductive health of the internal migrant population in China.

Supplementary Materials: The following are available online at http:/ / www.mdpi.com/1660-4601/16/4/655/s1, Table S1: Socio-demographic characteristics variables, Table S2: RTI-related knowledge, attitude, and practice variables, Table S3: Laboratory-confirmed RTIs species, Table S4: Internal migrant population of reproductive age from the Sixth Nationwide Population Census.

Author Contributions: Conceptualization, S.X.; data curation, Y.Z., J.W. and Y.L.; formal analysis, S.X.; funding acquisition, J.W.; investigation, S.X., Y.Z., J.W., T.B., J.Z., and Y.L.; methodology, S.X.; project administration, J.W.; resources, J.W.; software, S.X.; supervision, S.X. and J.W.; writing-original draft, S.X.; writing-review and editing, Y.L.

Funding: This project was funded by the National 12th Five-Year Plan (No.2012BAI32B08).

Acknowledgments: The authors are grateful to all the project investigators, the Family Planning and Reproductive Health Instructing Center of Ningxia Hui Autonomous Region, the Family Planning Publicity and Education Technical Advising Center of Urumchi, and the Songjiang Branch of Shanghai First People's Hospital staff for their cooperation and support. We also thank the study participants for their understanding and patience. 
Conflicts of Interest: There are no conflicts of interest to declare.

\section{References}

1. Newman, L.; Rowley, J.; Vander, H.S.; Wijesooriya, N.S.; Unemo, M.; Low, N.; Stevens, G.; Gottlieb, S.; Kiarie, J.; Temmerman, M. Global Estimates of the Prevalence and Incidence of Four Curable Sexually Transmitted Infections in 2012 Based on Systematic Review and Global Reporting. PLoS ONE 2015, 10, e143304. [CrossRef] [PubMed]

2. Koumans, E.H.; Sternberg, M.; Bruce, C.; McQuillan, G.; Kendrick, J.; Sutton, M.; Markowitz, L.E. The prevalence of bacterial vaginosis in the United States, 2001-2004; associations with symptoms, sexual behaviors, and reproductive health. Sex. Transm. Dis. 2007, 34, 864-869. [CrossRef] [PubMed]

3. Centers for Disease Control and Prevention. Genital/Vulvovaginal Candidiasis 2014. Available online: http:/ / www.cdc.gov / fungal/diseases/candidiasis/genital/index.html (accessed on 4 November 2016).

4. Powell, K. Bacterial vaginosis: Risks and treatment. Br. J. Nurs. 2013, 22, 1044. [CrossRef] [PubMed]

5. Huang, Y.Y.; Pan, S.M.; Wang, N. Self-reported STDs among Chinese adults: Prevalence and its related factors. Chin. J. Aids Std. 2012, 2, 69-72.

6. Donald, W.H. The changing pattern of sexually transmitted diseases in adolescents. Practitioner 1979, 222, 383-385.

7. Huang, Z.L.; Yi, L.; Wu, S.F.; Yang, G.L. Current situation investigation and intervention measures of reproductive tract infections of married women at childbearing age of community floating population. Chin. Community Dr. 2015, 29, 158-159.

8. Jiang, R.G.; Fu, X.; Zhou, J.; Liu, H.Y. Analysis on the status and the effect factors of reproductive tract infection of married women of child-Bearing age among floating population. Mater. Child Health Care China 2011, 26, 1825-1828.

9. Jin, M.P.; Tao, Y.C. The status and influencing factors of Reproductive Tract Infections of married floating population in Pinghu city. China Mod. Dr. 2011, 49, 147-149.

10. Zhang, J.X.; Zhou, Y.L.; Liu, J.S.; Lu, P.; Li, L.; Mei, J.; Tajiguli, T. Family Planning Publicity \& Education Technical Advising Center of Urumchi; Youyi Hospital in Urumchi, Xinjiang Uygur Autonomous Region. The prevalence rate and influencing factors of reproductive tract infections among floating women of childbearing age in Urumchi. Chin. J. Fam. Plann. 2014, 22, 666-670.

11. Zhang, L.J.; Geng, Y.T.; An, H.Y.; Gao, S.Y.; Zhang, L.; Cen, Y.; Zeng, G. [Investigation on reproductive tract infection among floating married women of childbearing age in Fengtai district in Beijing]. Zhonghua Liu Xing Bing Xue Za Zhi 2003, 24, 678-680.

12. Lin, W.; Huang, M.; Zhu, R.Y.; Li, J.R.; Wu, X.E.; Lin, Z.J.; You, A. Survey on the Status and Requirement of the Reproductive Health of Floating Population in Taizhou City. Chin. J. Fam. Plann. 2008.

13. Lou, C.H.; Shen, Y.; Gao, E.S.; Tu, X.W. Sexual Behaviors of Unmarried Migrants with Different Characteristics. Reprod. Contracept. 2005, 25, 738-744.

14. Kaliyaperumal, K. Guideline for Conducting a Knowledge, Attitude and Practice (KAP) Study. AECS Illum. 2004, 4, 7-9.

15. Patel, D.A.; Burnett, N.M.; Curtis, K.M.; Hillis, S.; Marchbanks, P. Reproductive Tract Infections; United States Department of Health and Human Services, Centers for Disease Control and Prevention: Atlanta, GA, USA, 2003.

16. Wasserheit, J.N.; Holmes, K.K. Reproductive Tract Infections: Global Impact and Priorities for Women's Reproductive Health; Plenum Press: New York, NY, USA, 1992.

17. Xie, Y. Demography: Past, Present, and Future. J. Am. Stat. Assoc. 2000, 95, 670-673. [CrossRef]

18. Zahedi, L.; Sizemore, E.; Malcolm, S.; Grossniklaus, E.; Nwosu, O. Knowledge, Attitudes and Practices Regarding Cervical Cancer and Screening among Haitian Health Care Workers. Int. J. Environ. Res. Public Health 2014, 11, 11541-11552. [CrossRef] [PubMed]

19. Lan, P.T.; Lundborg, C.S.; Phuc, H.D.; Sihavong, A.; Unemo, M.; Chuc, N.T.K.; Khang, T.H.; Mogren, I. Reproductive tract infections including sexually transmitted infections: A population-based study of women of reproductive age in a rural district of Vietnam. Sex. Transm. Infect. 2008, 84, 126-132. [CrossRef] [PubMed]

20. Yang, H.L.; Wu, J.Q.; Li, Y.Y.; Tong, Q. Prevalence and related factors of reproductive tract infection in floating population. Chin. J. Public Health 2016, 32, 73-75. 
21. Wu, Y.H.; Qian, L.H.; Feng, R.; Li, H.Y.; Qiu, X.Q. Analysis of the Reproductive Health Status of the Spinsterhood Girls in the Floating Population in Nanshan Distric to Shenzhen City. Chin. J. Fam. Plan. 2007, 15, 545-547.

22. Bhilwar, M.; Lal, P.; Sharma, N.; Bhalla, P.; Kumar, A. Prevalence of reproductive tract infections and their determinants in married women residing in an urban slum of North-East Delhi, India. J. Nat. Sci. Biol. Med. 2015, 6, S29-S34. [CrossRef]

23. Xu, C.Y.; Zhang, W.Y.; Wu, M.H.; Zhang, S.W. Prevalence and risk factors of lower genital tract infections among women in Beijing, China. J. Obstet. Gynaecol. Res. 2012, 38, 310-315.

24. Mao, Y.Y.; Cai, J.H.; Hu, Y.; Yu, H.; Zhou, W.J.; Che, Y. Male's Reproductive Tract Infections in 9 Provinces of China: Self-reported Prevalence and Determinants. Reprod. Contracept. 2010, 30, 187-192.

25. McGrath, N.; Eaton, J.; Newell, M.; Hosegood, V. Migration, sexual behaviour, and HIV risk: A general population cohort in rural South Africa. Lancet Hiv. 2015, 2, e252-e259. [CrossRef]

26. Shendre, M.C.; Tiwari, R.R. Role of occupation as a risk factor for sexually transmitted disease: A case control study. Indian J. Occup. Environ. Med. 2005, 9, 35-37.

27. Xie, X.; Gou, W.L. Obstetrics and Gynecology, 8th ed.; People's Medical Publishing House: Beijing, China, 2013.

28. Capoccia, R.; Greub, G.; Baud, D. Ureaplasma urealyticum, Mycoplasma hominis and adverse pregnancy outcomes. Curr. Opin. Infect. Dis. 2013, 26, 231-240. [CrossRef] [PubMed]

29. Huang, C.; Long, X.; Jing, S.; Fan, L.; Xu, K.; Wang, S.; Zhu, W. Ureaplasma urealyticum and Mycoplasma hominis infections and semen quality in 19,098 infertile men in China. World J. Urol. 2016, 34, 1039-1044. [CrossRef] [PubMed]

30. Wang, Y.; Wu, Z.W.; Zhang, L.F.; Wu, X.K.; Yi, L.; Han, X. Effects of Ureaplasma urealyticum infection on the male reproductive system in experimental rats. Andrologia 2010, 42, 297-301. [CrossRef] [PubMed]

31. Kataoka, S.; Yamada, T.; Chou, K.; Nishida, R.; Morikawa, M.; Minami, M.; Yamada, H.; Sakuragi, N.; Minakami, H. Association between preterm birth and vaginal colonization by mycoplasmas in early pregnancy. J. Clin. Microbiol. 2006, 44, 51-55. [CrossRef]

32. Abusarah, E.A.; Awwad, Z.M.; Charvalos, E.; Shehabi, A.A. Molecular detection of potential sexually transmitted pathogens in semen and urine specimens of infertile and fertile males. Diagn. Microbiol. Infect. Dis. 2013, 77, 283-286. [CrossRef] [PubMed]

33. Lan, P.T.; Faxelid, E.; Chuc, N.T.; Mogren, I.; Lundborg, C.S. Perceptions and attitudes in relation to reproductive tract infections including sexually transmitted infections in rural Vietnam: A qualitative study. Health Policy 2008, 86, 308-317. [CrossRef] [PubMed]

34. Sheeran, P.; Maki, A.; Montanaro, E.; Avishai-Yitshak, A.; Bryan, A.; Klein, W.M.P.; Miles, E.; Rothman, A.J. The impact of changing attitudes, norms, and self-efficacy on health-related intentions and behavior: A meta-analysis. Health Psychol. 2016, 35, 1178-1188. [CrossRef] [PubMed]

35. Aral, S.O.; Fenton, K.A.; Lipshutz, J.A. The New Public Health and STD/HIV Prevention: Personal, Public and Health Systems Approaches; Springer Science \& Business Media: New York, NY, USA, 2012.

36. Hancock, E.B.; Manhart, L.E.; Nelson, S.J.; Kerani, R.; Wroblewski, J.K. Comprehensive assessment of sociodemographic and behavioral risk factors for Mycoplasma genitalium infection in women. Sex. Transm. Dis. 2010, 37, 777-783. [CrossRef] [PubMed]

37. Luo, L.; Xu, J.; Wang, G.; Ding, G.; Wang, N.; Wang, H. Vaginal douching and association with sexually transmitted infections among female sex workers in a prefecture of Yunnan Province, China. Int. J. STD AIDS 2016, 27, 560-567. [CrossRef] [PubMed]

38. China Central Television (CCTV). Weekly Quality Report: Wipes Security. 2015. Available online: http: / / tv.cntv.cn/video/C10354/c303ab0d12834757ab89a31dccf10ce2 (accessed on 22 May 2017).

39. Centers for Disease Control Prevention. Condoms and STDs: Fact Sheet for Public Health Personnel 2013. Available online: https:/ / www.cdc.gov / condomeffectiveness/latex.html (accessed on 2 February 2018).

40. World Health Organization. Family planning/Contraception. 2016. Available online: http://www.who.int/ mediacentre/factsheets/fs351/en/ (accessed on 21 May 2017).

41. World Health Organization. Sexually Transmitted Infections (STIs). 2016. Available online: http:/ / www. who.int/mediacentre/factsheets/fs110/en/ (accessed on 21 May 2017).

42. World Health Organization. Sexually Transmitted and Other Reproductive Tract Infections: A Guide to Essential Practice; Department of Reproductive Health and Research, World Health Organization: Geneva, Switzerland, 2005. 
43. Baeten, J.M.; Nyange, P.M.; Richardson, B.A.; Lavreys, L.; Chohan, B.; Martin, H.L.; Mandaliya, K.; Ndinya-Achola, J.O.; Bwayo, J.J.; Kreiss, J.K. Hormonal contraception and risk of sexually transmitted disease acquisition: Results from a prospective study. Am. J. Obstet. Gynecol. 2001, 185, 380-385. [CrossRef] [PubMed]

44. Borgdorff, H.; Verwijs, M.C.; Wit, F.W.; Tsivtsivadze, E.; Ndayisaba, G.F.; Verhelst, R.; Schuren, F.H.; van de Wijgert, J.H. The impact of hormonal contraception and pregnancy on sexually transmitted infections and on cervicovaginal microbiota in african sex workers. Sex. Transm. Dis. 2015, 42, 143-152. [CrossRef] [PubMed]

45. Rifkin, S.B.; Smith, M.R.; Brotman, R.M.; Gindi, R.M.; Erbelding, E.J. Hormonal contraception and risk of bacterial vaginosis diagnosis in an observational study of women attending STD clinics in Baltimore, MD. Contraception 2009, 80, 63-67. [CrossRef] [PubMed]

46. Murphy, K.; Mitchell, C.M. The Interplay of Host Immunity, Environment and the Risk of Bacterial Vaginosis and Associated Reproductive Health Outcomes. J. Infect. Dis. 2016, 214, S29-S35. [CrossRef] [PubMed]

47. Panchanadeswaran, S.; Johnson, S.C.; Mayer, K.H.; Srikrishnan, A.K.; Sivaram, S.; Zelaya, C.; Go, F.; Solomon, S.; Bentley, M.E.; Celentano, D.D. Gender differences in the prevalence of sexually transmitted infections and genital symptoms in an urban setting in southern India. Sex. Transm. Infect. 2006, 82, 491-495. [CrossRef]

48. Nielsen, A.; Lan, P.T.; Marrone, G.; Phuc, H.D.; Chuc, N.T.; Stalsby, L.C. Reproductive Tract Infections in Rural Vietnam, Women's Knowledge, and Health-Seeking Behavior: A Cross-Sectional Study. Health Care Women Int. 2016, 37, 392-411. [CrossRef] [PubMed]

49. Valente, A.M.; Auerswald, C.L. Gender differences in sexual risk and sexually transmitted infections correlate with gender differences in social networks among San Francisco homeless youth. J. Adolesc. Health 2013, 53, 486-491. [CrossRef] [PubMed]

(C) 2019 by the authors. Licensee MDPI, Basel, Switzerland. This article is an open access article distributed under the terms and conditions of the Creative Commons Attribution (CC BY) license (http:/ / creativecommons.org/licenses/by/4.0/). 